\title{
Usefulness of the soluble membrane attack complex to determine dermatomyositis clinical activity and treatment response
}

\author{
Andrés Tirado-Sánchez, Alexandro Bonifaz and Rosa María Ponce-Olivera \\ Secretaría de Salud, Hospital General de México, Dermatology Department, Ciudad de México, Mexico
}

\begin{abstract}
Introduction: Dermatomyositis is an autoimmune disease and the most common idiopathic inflammatory myopathy. During patient follow-up, determining biochemical parameters is required in order to assess for disease activity and treatment efficacy. Objective: To determine the relationship between the degree of activation of the complement system through the soluble membrane attack complex (c5b-9), dermatomyositis clinical activity and its variations with conventional treatment. Method: Forty-five patients with active and inactive dermatomyositis were studied. Chemical parameters and clinical severity were established and correlated with ELISA-determined C5b-9 serum levels. Results: There was positive correlation between dermatomyositis cutaneous and muscular activity and C5b-9 serum levels, which was lower than with traditional biochemical markers. In the case of treatment response, C5b-9 showed significant reduction, similar to clinical severity; with biochemical parameters, the reduction was not significant at one month of treatment with systemic steroids. Conclusions: Serum levels of C5b-9 levels of C5b-9 are higher in patients with dermatomyositis than in healthy subjects; dermatomyositis active and inactive cases were determined by means of their measurement. They can be a reliable parameter of therapeutic response, more accurate than muscle enzymes measurement, particularly creatine phosphokinase.
\end{abstract}

KEY WORDS: Dermatomyositis. Activity. Complement system. C5b-9. Creatine phosphokinase.

\section{Introduction}

Dermatomyositis is an autoimmune disease included in the group of idiopathic inflammatory myopathies. ${ }^{1}$ It is a disease with common complications deriving from visceral involvement or from progression or exacerbation of the process. ${ }^{2}$ In recent decades, attempts have been made to identify serological markers that facilitate dermatomyositis diagnosis, ${ }^{3}$ and that also assess clinical response to different treatment regimens and, as a consequence, that allow prognosis of the disease to be established.

The parameters used for diagnosis (muscular strength, muscle enzymes, histological findings and electromyography), although useful, are not sensitive or specific enough to assess treatment response; ${ }^{3}$ therefore, other options related to the pathogenesis of the disease have been studied in order for clinical correlation to be established (for example, associated with muscle injury, with endothelial lesion, with the immune phenomenon, with cytokines, with adhesion molecules, among others); however, none has obtained a sufficient degree of correlation to replace traditionally used biochemical and electrophysiological markers. ${ }^{2,3}$

Muscle damage in dermatomyositis has been observed to be related to complement-mediated microangiopathy, ${ }^{4,5}$ with membrane attack complex or C5b-9 deposits being described in the muscular microvasculature of patients with the disease, ${ }^{5}$ at different degrees of severity and with positive gradient, with its potential usefulness being translated into determining the degree of disease activity.

C5b-9 is a complex of proteins that bind to each other to form pores through the membrane, which results in cell death. ${ }^{6-8}$ Several studies have shown deposits of this protein complex in the skin, muscle fibers and in arterioles
Correspondence:

Andrés Tirado-Sánchez

E-mail: atsdermahgm@gmail.com
Date of reception: 09-08-2017

Date of acceptance: 27-07-2018

DOI://dx.doi.org/10.24875/GMM.M18000193
Gac Med Mex. 2018;154:493-497

Contents available at PubMed www.gacetamedicademexico.com 
and capillaries in biopsies of patients with dermatomyositis. ${ }^{9-11}$ The measurement of complement activation product levels is a measure of the catabolic state and, consequently, it is more accurate for establishing the degree of activation thereof.

So far, there are only few studies focused on the analysis of patients with dermatomyositis and, for this reason, we conducted an investigation to determine the relationship between the degree of activation of the complement system through the C5b-9 soluble complex, dermatomyositis clinical activity and its variations with the treatment.

\section{Methods}

The study was carried out between 2005 and 2011 and was approved by the Committee of Ethics in Research with registry code DI-06-109-3-61. All patients and healthy controls gave their consent to participate in the study. Forty-five patients of the female gender between 28 and 46 years of age and who met Bohan and Peter ${ }^{12,13}$ criteria were included:

- Group 1: patients with recent diagnosis, untreated and with active disease.

- Group 2: patients with inactive disease, with or without maintenance treatment.

A group of healthy subjects matched by age with the two previous groups in a 1: 1 ratio was included.

Patients were assessed at the moment of inclusion to the study, always by the same researcher. Disease severity was determined using data obtained from the medical records, by interrogation and physical examination using the Disease Activity Evaluation Scale (MYOACT), established by the International Myositis Assessment and Clinical Studies Group. ${ }^{14}$ The MYOACT score was recorded as muscular, cutaneous and total. Patients with overlap syndrome or dermatomyositis varieties other than type 2 were excluded.

Patients considered with inactive disease were to maintain a score of 0 on MYOACT (muscle, cutaneous and total) for at least one month. Patients who did not meet this criterion were considered to have active disease.

Clinical data were obtained when the patient agreed to participate in the study and included age, time of disease evolution and the MYOACT score in its three modalities (cutaneous, muscular and total). Blood samples were obtained $(10 \mathrm{~mL})$ for the measurement of creatine phosphokinase as a biochemical marker of dermatomyositis, as well as serum C5b-9. For the controls, blood samples were obtained from 45 healthy women, matched by age ( 26 to 45 years of age), from the hospital blood bank. The obtained blood sample was used to determine creatine phosphokinase and C5b-9 serum concentration by the ELISA technique, using a commercial kit of human serum C5b-9 (MyBiosource, San Diego, CA, USA). All samples, both from cases and controls, were simultaneously analyzed and in duplicate, with patient clinical status and identity being unknown. The definitive value of levels for each case was obtained by calculating the mean of the two obtained values.

After the sample was taken in patients with active disease, treatment was started with prednisone at $1 \mathrm{mg} / \mathrm{kg} / \mathrm{day}$ for 4 weeks, with new blood sample collection.

Statistical analysis was carried out with Fisher's exact test for categorical variables' comparison and Mann-Whitney rank test for comparison of numerical variables. Correlation coefficients were established with Spearman's correlation. To compare quantitative variables averages, Student's t-test (between groups) and paired t-tests (before and after) were used. Data were analyzed with SPSS, version 22.0. A p-value $<0.05$ was considered to be statistically significant.

\section{Results}

Patient clinical and biochemical characteristics are presented in table 1. Overall mean disease duration was 8 months (range: 2 to 17 months). At study inclusion, 28 patients had active disease (group 1) and 17 inactive disease (group 2).

Serum C5b-9 concentration values, as well as creatine phosphokinase levels are shown in table 1. Significant differences are observed in C5b-9 levels between cases with active disease and those with inactive disease $(p=0.008)$ and controls $(p=0.001)$ (Fig. 1). Creatine phosphokinase levels also showed significant differences between the three groups.

Serum C5b-9 correlation coefficients with disease severity yielded a positive correlation, not so with creatine phosphokinase levels; similarly, the latter parameter showed low correlation with clinical severity (Table 2).

Twenty-eight patients newly diagnosed with dermatomyositis were treated with prednisone at $1 \mathrm{mg} / \mathrm{kg} / \mathrm{day}$. Serum C5b9 levels at the beginning of the study $(67.45 \pm 18.35)$ were significantly higher than those measured at the conclusion of treatment $(17.33 \pm 8.43$, $p=0.001$ ) (Fig. 2). Creatine phosphokinase levels did not show significant reduction at one month of treatment, although clinical parameters of activity (MYOACT) had a significant reduction after treatment with systemic steroids (Table 3 ). 
Table 1. Clinical characteristics of patients with dermatomyositis

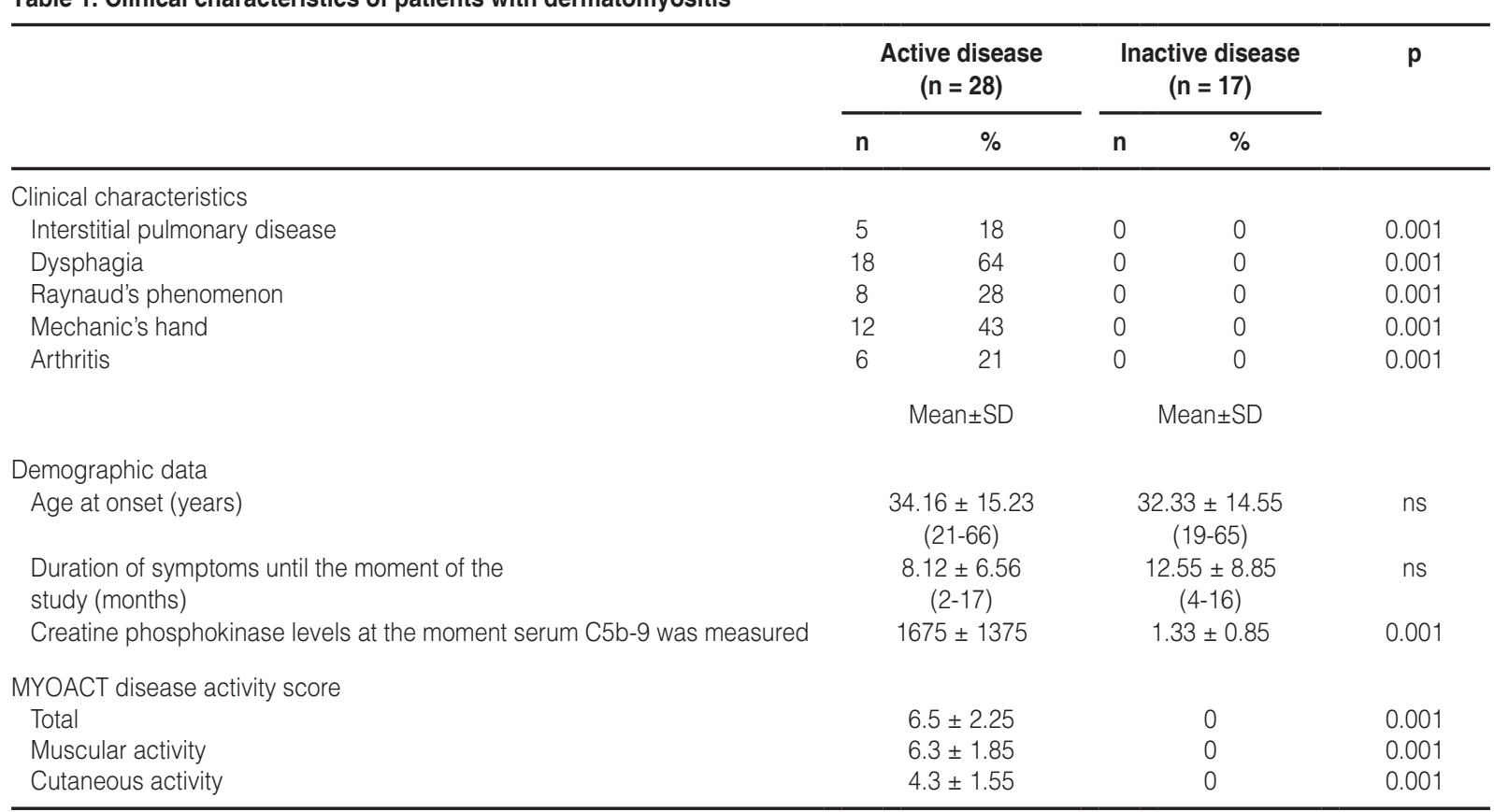

MYOACT = Myositis Disease Activity Assessment, SD = standard deviation, ns = not significant.

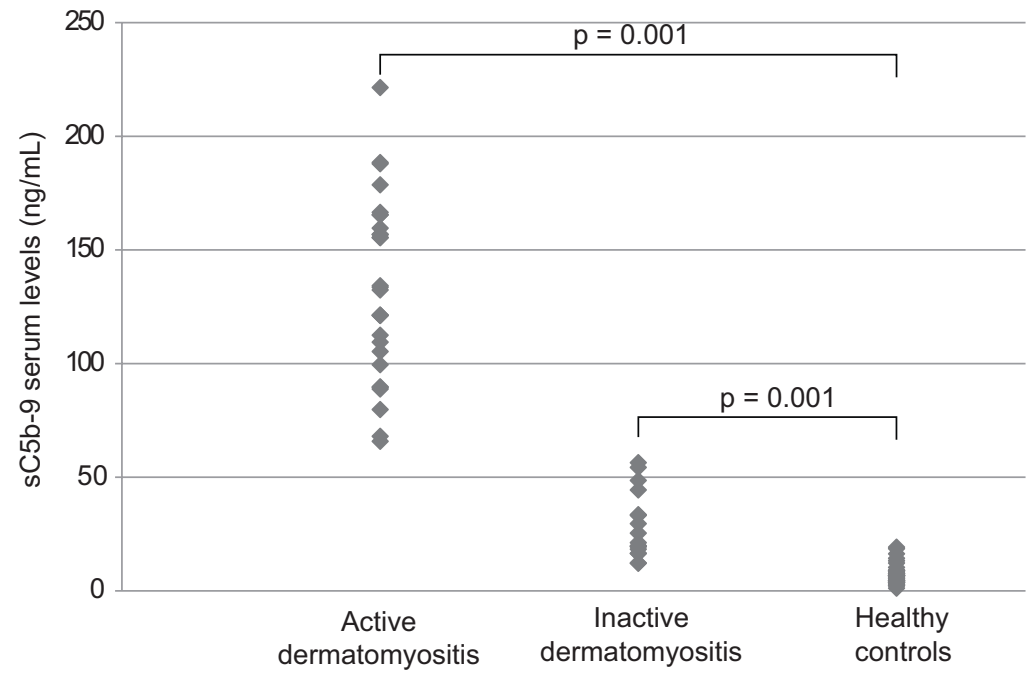

Figure 1. sC5b-9 serum levels in patients with dermatomyositis with active and inactive disease and in healthy subjects.

\section{Discussion}

C5b-9 serum levels in patients with dermatomyositis were higher than in healthy subjects; in addition, determining dermatomyositis active and inactive cases was possible with this parameter. Another important aspect is that serum C $5 \mathrm{~b}-9$ can be a reliable parameter of therapeutic response, even more accurate than measuring muscle enzymes, particularly creatine phosphokinase.

Elevated serum C5b-9 levels in patients with dermatomyositis confirm complement activation in the pathogenesis of the disease and tend to be higher in patients with active rather than inactive disease and in healthy controls. In a previous work by Campo et al.,., ${ }^{11}$ C5b-9 serum levels were observed to be correlated with disease severity, although the results didn't allow to evaluate treatment response, even when it is possible to distinguish patients with active and inactive disease. In our study, in addition to observing a good correlation between clinical activity and biochemistry of the disease and the marker, as well as its ability to distinguish between active and inactive disease, it was useful to assess treatment response, and thus it could be useful to determine the prognosis. 
Table 2. Correlation between C5b-9 serum levels, creatine phosphokinase and clinical markers in patients with dermatomyositis

\begin{tabular}{lccc}
\hline & C5b-9 levels & Creatine phosphokinase & Total MYOACT \\
\hline C5b-9 levels & 1 & $0.122, p=0.063$ & $0.433, p=0.001$ \\
Creatine phosphokinase & $0.122, p=0.063$ & 1 & $0.231, p=0.05$ \\
Total MYOACT & $0.433, p=0.001$ & $0.231, p=0.05$ & 1 \\
\hline Correlations were performed with Pearson's test; statistical significance with $p<0.05$. MYOACT $=$ clinical markers. &
\end{tabular}

Table 3. C5b-9 serum concentration, creatine phosphokinase and MYOACT before and after one-month treatment with systemic steroids in patients with dermatomyositis

\begin{tabular}{lcclc}
\hline Variable & Before treatment & One month after treatment & $\mathbf{p}$ \\
\hline C5b-9 serum levels $(\mathrm{ng} / \mathrm{mL})$ & $67.45 \pm 18.35$ & $17.33 \pm 8.43$ & $1427 \pm 1239$ & 0.001 \\
Creatine phosphokinase $(\mathrm{mg} / \mathrm{dL})$ & $1675 \pm 1375$ & $1.7 \pm 1.3$ & 0.049 \\
Total MYOACT & $6.5 \pm 2.25$ & 0.001 \\
\hline
\end{tabular}

MYOACT = Myositis Disease Activity Assessment

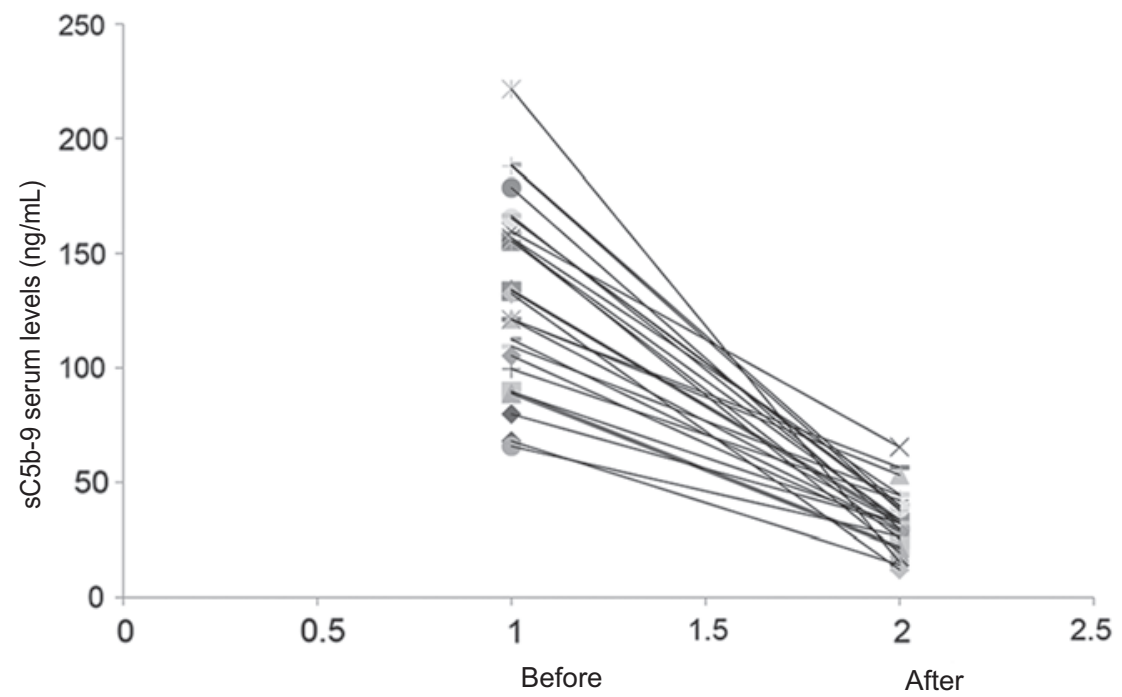

Figure 2. sC5b-9 serum levels after one month of treatment with systemic steroids.

C5b-9 is an inflammatory mediator related to the pathophysiology of many diseases. Evidences show that C5b-9 is an indicator of clinical severity of diseases it has been associated to, including systemic lupus erythematosus and dermatomyositis and, therefore, blocking this inflammatory mediator in many inflammatory disease models contributes to reduce its severity. ${ }^{15}$

In our study, we observed a positive correlation between C5b-9 serum levels and signs of disease activity and, similar to observations reported by Campo et al., ${ }^{11}$ this complement activation parameter showed no correlation with traditional activity markers, specifically creatine phosphokinase.

In dermatomyositis, muscle enzymes are released as a result of muscle fiber lesion, and thus the temporal correlation between their increase and the development of muscle weakness is important. In C5b-9 serum levels, elevation can precede muscular activity and possibly cutaneous activity, and its levels are maintained during disease activity and are correlated with severity.

The primary antigenic target in dermatomyositis is the endomysium capillaries endothelium. These antibodies elicit C3 activation, which in turn causes the formation of C3b, C4b and of C5b-9 fragments, which are detectable in the course of the disease both in serum and the capillaries, and are even observed in muscle tissue prior to inflammatory or structural changes. These deposits are important to induce muscle fiber destruction. ${ }^{96}$

Another mechanism to explain the relationship between C5b-9 and the development of dermatomyositis is based on membrane attack complex activation, which causes an excess of $\mathrm{Ca}^{2+}$ that gives rise to mitochondrial overload and mitochondrial transmembrane potential loss, thus resulting in an energy crisis within the cells, while ion pumps try to 
reestablish $\mathrm{Ca}^{2+}$ homeostasis; cytochrome $\mathrm{C}$ release into the cytoplasm and caspase activation trigger apoptosis This suggests the crucial role of C5b-9 in NALP3 inflammasome activation, by means of which it amplifies the inflammatory response. ${ }^{17,18}$ The NLRP3 inflammasome plays a key role in the development of dermatomyositis, ${ }^{19}$ which demonstrates that C5b-9 activation may precede the development of clinical, biochemical and immune manifestations of the disease.

One limitation of our study is that it was not possible to compare C5b-9 serum levels with skin and muscle deposits thereof, which is why our study could serve as a basis for others to assess whether such a correlation exists. We assume it does, since, in previous studies, muscle biopsies of patients with dermatomyositis were observed to show C5b-9 deposits in endothelial cells. ${ }^{20}$ In addition, C5b-9 deposits were observed in injured skin biopsies of patients with the disease, not so on unaffected skin, suggesting that it would also be involved in the pathogenesis of skin lesions. ${ }^{21}$

On the other hand, given that this study included one single center, the findings require confirmation in other hospitals.

Current treatments against the complement, especially eculizumab, specifically targeting C5b-9 or alternate pathways, are under development. ${ }^{15}$

In conclusion, complement system activation product levels quantification is a tool for clinical control of patients with dermatomyositis, since it allows to distinguish between active and inactive disease and adequately correlate with treatment response.

On the other hand, it is necessary to determine whether C5b-9 serum levels might serve to distinguish other types of myositis.

\section{References}

1. Findlay AR, Goyal NA, Mozaffar T. An overview of polymyositis and dermatomyositis. Muscle Nerve 2015;51:638-656.
2. Luo YB, Mastaglia FL. Dermatomyositis, polymyositis and immune-mediatednecrotising myopathies. Biochim Biophys Acta. 2015;1852:622-632.

3. Iaccarino L, Ghirardello A, Bettio S, Zen M, Gatto M, Punzi L, et al. The clinical features, diagnosis and classification of dermatomyositis. J Autoimmun. 2014;48-49:122-127.

4. Lahoria R, Selcen D, Engel AG. Microvascular alterations and the role of complement in dermatomyositis. Brain. 2016;139(Pt 7):1891-1903.

5. Kissel JT, Halterman RK, Rammohan KW, Mendell JR. The relationship of complement-mediated microvasculopathy to the histologic features and clinical duration of disease in dermatomyositis. Arch Neurol. 1991;48:26-30.

6. Serna M, Giles JL, Morgan BP, Bubeck D. Structural basis of complementmembrane attack complex formation. Nat Commun. 2016;7:10587.

7. Dudkina NV, Spicer BA, Reboul CF, Conroy PJ, Lukoyanova N, Elmlund $\mathrm{H}$, et al. Structure of the poly-C9 component of the complement membrane attack complex. Nat Commun. 2016;7:10588.

8. Bayly-Jones C, Bubeck D, Dunstone MA. The mystery behind membrane insertion: a review of the complement membrane attack complex. Philos Trans R Soc Lond B Biol Sci. 2017;372:20160221.

9. Braczynski AK, Harter PN, Zeiner PS, Drott U, Tews DS, Preusse C, et al. C5b-9 deposits on endomysial capillaries in non-dermatomyositis cases. Neuromuscul Disord. 2016;26:283-291.

10. Sakuta R, Murakami N, Jin Y, Nagai T, Nonaka I, Nishino I. Diagnostic significance of membrane attack complex and vitronectin in childhood dermatomyositis. J Child Neurol. 2005;20:597-602.

11. Campo A, Hausmann G, Martí RM, Estrach T, Grau JM, Porcel JM, et al. Complement activation products ( $\mathrm{C} 3 \mathrm{a}$ and $\mathrm{C} 5 \mathrm{~b}-9)$ as markers of activity ofdermatomyositis. Comparison with usual biochemical parameters. Actas Dermosifiliogr. 2007;98:403-414.

12. Bohan A, Peter JB. Polymyositis and dermatomyositis (second of two parts). N Engl J Med. 1975;292:403-407.

13. Bohan A, Peter JB. Polymyositis and dermatomyositis (first of two parts). N Engl J Med 1975;292(7):344-7.

14. Isenberg DA, Allen E, Farewell V, Ehrenstein MR, Hanna MG, Lundberg IE, et al. International consensus outcome measures for patients with idiopathic inflammatory myopathies. Development and initial validation of myositis activity and damage indices in patients with adult onset disease. Rheumatology (Oxford). 2004;43:49-54.

15. Morgan BP. The membrane attack complex as an inflammatory trigger. Immunobiology. 2016;221:747-751.

16. Ballanti E, Perricone C, Greco E, Ballanti M, Di-Muzio G, Chimenti MS, et al. Complement and autoimmunity. Immunol Res. 2013;56:477-491.

17. Triantafilou K, Hughes TR, Triantafilou M, Morgan BP. The complement membrane attack complex triggers intracellular $\mathrm{Ca} 2+$ fluxes leading to NLRP3 inflammasome activation. J Cell Sci. 2013;126(Pt 13):2903-2913.

18. Zhao T, Gao J, Van J, To E, Wang A, Cao S, et al. Age-related increases in amyloid beta and membrane attack complex: evidence of inflammasome activation in the rodent eye. J Neuroinflammation. 2015;12:121.

19. Yin $X$, Han GC, Jiang $X W$, Shi $Q, P u C Q$. Increased expression of the NOD-like receptor family, pyrin domain containing 3 inflammasome in dermatomyositis and polymyositis is a potential contributor to their pathogenesis. Chin Med J (Engl). 2016;129:1047-1052.

20. Kissel JT, Mendell JR, Rammohan KW. Microvascular deposition of complement membrane attack complex in dermatomyositis. $\mathrm{N}$ Engl $\mathrm{J}$ Med. 1986;314:329-334.

21. Máscaro JM, Hausmann G, Herrero C, Grau JM, Cid MC, Palou J, et al. Membrane attack complex deposits in cutaneous lesions of dermatomyositis. Arch Dermatol. 1995;131:1386-1392. 